Relations industrielles

Industrial Relations

\title{
Barbier, Jean-Claude et Jérôme Gautié (dir.), Les politiques de l'emploi en Europe et aux Éats-Unis
}

\section{Diane Bellemare}

Volume 54, numéro 4, 1999

URI : https://id.erudit.org/iderudit/051281ar

DOI : https://doi.org/10.7202/051281ar

Aller au sommaire du numéro

Éditeur(s)

Département des relations industrielles de l'Université Laval

ISSN

0034-379X (imprimé)

1703-8138 (numérique)

Découvrir la revue

Citer ce compte rendu

Bellemare, D. (1999). Compte rendu de [Barbier, Jean-Claude et Jérôme Gautié (dir.), Les politiques de l'emploi en Europe et aux Éats-Unis]. Relations industrielles / Industrial Relations, 54(4), 830-834.

https://doi.org/10.7202/051281ar

Tous droits réservés @ C Département des relations industrielles de l'Université Laval, 1999
Ce document est protégé par la loi sur le droit d'auteur. L’utilisation des services d'Érudit (y compris la reproduction) est assujettie à sa politique d'utilisation que vous pouvez consulter en ligne.

https://apropos.erudit.org/fr/usagers/politique-dutilisation/ 
it happened, though the debate on why and who gained still continues. That sort of analysis and focus on the broad picture of living standards in the US is what makes this book so valuable. The authors systematically and carefully document both the good and the bad in the economy and keeps its eye on the big economic issues while arguing with solid statistics. But perhaps one of the most useful features of the book is simply its accessibility. It is not written only for economists and it doesn't overpower with econometric technique when a simple table will make the point.

Throughout The State of Working America, it is clear that the authors' per- spective sits to the left of the economic establishment in the US, but in writing such an accessible book, they encourage increased economic literacy among people from across the political spectrum. Additionally, their international comparisons may help inform debate and policy making among those OECD countries that are looking to the US model for solutions to their labour market problems.

LAURA DRESSER

Center on Wisconsin Strategy University of Wisconsin-Madison

\section{Les politiques de l'emploi en Europe et aux États-Unis}

sous la direction de Jean-Claude BARBIER et Jérôme GAuTIÉ, Paris : Presses universitaires de France, 1998, 435 p., ISBN 2-11-088609-9.

La lecture de ce livre est fortement recommandée pour quiconque s'intéresse de près ou de loin aux politiques de l'emploi. Il se divise en deux parties. On y retrouve d'abord une description des principaux points qui caractérisent les politiques de l'emploi dans plusieurs pays européens parmi lesquels on retrouve le Royaume-Uni, la France, la République fédérale d'Allemagne, la Suède, le Danemark, les Pays-Bas ainsi que les politiques d'emploi américaines. Ensuite, le livre présente des analyses transversales effectuées par différents auteurs qui évaluent, notamment, les impacts de ces politiques dans une perspective microéconomique et macro-économique et qui se penchent également sur des questions d'intérêt général comme le partage du travail, l'économie sociale et la recherche d'une politique européenne de l'emploi. Le livre conclut en s'interrogeant, à partir des comparaisons de politiques nationales de l'emploi, sur le cadre d'analyse le plus approprié pour effectuer de telles comparaisons ainsi que sur l'avenir de telles politiques.

Rappelons que les politiques de l'emploi désignent généralement l'ensemble des interventions prises dans le cadre des politiques économique et sociale d'un pays pour influer sur le niveau de l'emploi et du chômage. Ces interventions peuvent être d'ordre budgétaire, monétaire, fiscal, réglementaire ou encore elles peuvent être plus ciblées et concernent généralement les politiques du marché du travail qui influent directement sur l'offre et la demande de travail ainsi que sur l'ajustement entre les deux.

La lecture de l'ouvrage conduit au constat suivant, à savoir que pour plusieurs pays, la politique de l'emploi n'en est qu'à ses balbutiements. Au contraire, la politique du marché du travail est développée et concrète dans tous les pays étudiés. À cet effet, il est intéressant de constater que la réduction et l'aménagement du temps de travail ont joué un rôle central dans le cadre de la politique de l'emploi de certains pays parmi lesquels on retrouve la République fédérale d'Allemagne, la France, les Pays-Bas. Dans ces pays, toutefois, même si la durée hebdomadaire légale a diminué depuis 20 ans, la forte baisse des heures annuelles moyennes rémunérées s'expliquerait en 
grande partie par la croissance du travail à temps partiel.

Plusieurs contributions dans la première partie du livre se concentrent sur les politiques du marché du travail et, en particulier, sur la question de l'activation des mesures passives en mesures actives. En effet, comme au Canada et au Québec, plusieurs pays consacrent une forte proportion des budgets alloués à la politique du marché du travail au soutien du revenu des personnes bénéficiant de l'assurance-emploi ou de l'assurancechômage et de l'aide sociale. Selon les dernières données de l'OCDE, le Canada consacrait en $199830 \%$ des dépenses sur le marché du travail pour les mesures actives, c'est-à-dire les mesures de formation, d'insertion, de création, de maintien et de stabilisation de l'emploi. Depuis déjà la fin des années 80 , nombre de pays tentent d'augmenter la proportion des mesures actives au lieu des mesures passives.

Les différentes contributions, à l'exception de celles concernant le RoyaumeUni et l'Espagne, s'attardent peu aux questions de la formation de la maind'œuvre. Bien que ces dépenses demeurent toujours importantes, l'intérêt des analystes porte davantage sur l'activation des mesures passives en mesure actives. Toutefois, le lecteur intéressé par les questions de formation professionnelle sera satisfait de lire comment, au Royaume-Uni, on tente de remédier au problème de l'insuffisance de la qualification de la main-d'œuvre par la mise en place d'un système de National Vocational Qualification (NVQ).

Dans la perspective de l'activation des mesures passives, plusieurs contributions (notamment celles concernant la République fédérale d'Allemagne, la France, les Pays-Bas) décrivent abondamment les mesures de subventions à l'emploi, lesquelles sont généralement importantes dans ces pays et destinées à des clientèles particulières comme les jeunes, les femmes monoparentales, les chômeurs de longue durée, les personnes handicapées ou d'autres groupes de personnes démunies. À titre d'exemple, on apprend qu'aux Pays-Bas, en 1997, 23000 jeunes de moins de 23 ans qui étaient au chômage travaillaient dans le cadre d'emplois subventionnés soit dans des municipalités, dans le secteur à but non lucratif et depuis 1995 dans des entreprises privées (p. 127). Ce chiffre est relativement élevé compte tenu qu'en 1997, la population des 15-64 ans était de 10563 millions de personnes.

On retrouve, dans cette première partie du livre, une brève explication des réformes des services publics d'emploi ainsi que des façons de faire dans le cadre des nouvelles relations de réciprocité qui lient le bénéficiaire et le service public d'emploi.

Plusieurs articles (principalement ceux concernant la République fédérale d'Allemagne, les Pays-Bas, le Danemark, la Suède, la Finlande) insistent sur la place des partenaires du marché du travail, que sont les représentants des salariés et des entreprises, dans le cadre de la politique de l'emploi et plus spécifiquement dans le cadre de la politique du marché du travail. Ainsi, le Danemark souligne le fait que l'une des conditions de succès des politiques de l'emploi réside dans la coordination des différents réseaux que sont les entreprises et les autorités régionales et locales, les agences pour l'emploi ainsi que les institutions chargées de la formation et que, dans cette perspective, les partenaires du marché du travail réunis dans des "Conseils du marché du travail régional " jouent un rôle déterminant en la matière (p. 171).

Les différentes analyses nationales foisonnent également d'une foule de renseignements qui peuvent être utiles pour stimuler l'imagination des décideurs publics. À titre d'exemple, on apprend que le Danemark a consacré, depuis 1994, en moyenne $0,3 \%$ de son PIB pour des mesures actives en faveur des personnes 
handicapées, soit $15 \%$ du total des dépenses consacrées aux mesures actives (p. 169).

On apprend également qu'aux ÉtatsUnis, la politique de l'emploi a été traditionnellement "définie en termes de politique sociale et, plus spécifiquement, de mesures de lutte contre la pauvreté " (p. 219). L'auteure (Sylvie Morel, professeur au Département des relations industrielles de l'Université Laval) explique que " considérées comme des "politiques d'aide sociale", les mesures d'emploi américaines étaient incapables de recueillir le soutien d'une large partie de la population étant donné que seules les personnes pauvres en bénéficiaient" (p. 220). Toutefois, une autre contribution sur les politiques d'emplois américaines explique le virage que le Department of Labor entreprend depuis les années 90 dans le but d'élargir les mesures d'emploi à l'ensemble des chômeurs et, plus spécifiquement, aux licenciés économiques (p. 211-212). Cet article est intéressant pour avoir une vue d'ensemble de la réforme américaine en cours visant à établir partout au pays des guichets uniques universels pour les mesures de maind'œuvre et d'emploi.

On est surpris, dans le cas de la France, par l'ampleur des dispositifs de la politique de l'emploi adoptés ces dernières années afin d'augmenter le nombre d'emplois. Ainsi, on y apprend qu'en 1996, 2817 millions de personnes ont participé dans les dispositifs de la politique de l'emploi, soit plus de $10 \%$ de la population active et que "en fin d'années 1997, quelque deux millions de personnes occupaient des emplois aidés dans le cadre de dispositifs spécifiques, soit $10 \%$ des vingt millions de salariés " (p. 71).

Les différentes contributions de cette première partie sont dans l'ensemble très bien documentées et d'actualité. Toutefois, la façon de traiter l'information statistique dans les différents articles ne permet pas au lecteur de comparer vraiment les politiques du marché du travail d'un pays à un autre.

La deuxième partie de l'ouvrage est plus analytique, méthodologique et théorique. On peut y lire, entre autres, deux articles, l'un portant sur les évaluations d'ordre micro-économique des politiques d'emploi sur les bénéficiaires, l'autre portant sur les impacts macro-économiques de ces mêmes politiques. Ces deux articles débutent par une discussion des problèmes méthodologiques et théoriques associés à ces évaluations et se terminent par une brève recension des principaux résultats obtenus. Le lecteur averti se verra conforté par les conclusions conformes à la littérature existante. Les autres seront surpris peutêtre d'apprendre que " certaines formations de type scolaire se révèlent peu adaptées pour des publics particulièrement désavantagés (comme des jeunes ou des chômeurs de longue durée sans qualification), qui ont connu la plupart du temps l'échec scolaire et qui risquent ainsi d'être renvoyés à cette situation d'échec *. Ou encore d'apprendre que "les aides et incitations à la recherche d'emploi sont souvent considérées comme les mesures ayant le meilleur impact au moindre coût " (p. 249). Plusieurs seront aussi surpris de lire que les effets des politiques d'emploi " sont plutôt décevants en ce qui concerne les chômeurs de longue durée, ce qui prouverait que, comme on l'a souligné plus haut, il faut intervenir assez tôt pour éviter que n'apparaissent des situations difficilement réversibles " (p. 251). Néanmoins, ces articles confirment les effets positifs des politiques d'emploi aux plans macroéconomique et micro-économique.

Dans l'ensemble, tous les articles de cette deuxième partie présentent un intérêt évident; ils soulèvent chacun des questions pertinentes et importantes dans un contexte de chômage massif. Par exemple, on y aborde, de manière globale, la question du partage du travail dans l'Union européenne ainsi que l'évolution des politiques d'emploi et des 
nouveaux services en Europe comme le développement de l'économie sociale.

On y retrouve aussi un article fort intéressant de Jean-Claude Barbier portant sur la nécessité d'analyser la mise en œuvre des politiques de l'emploi dans le cadre de l'évaluation des politiques. Selon l'auteur, trop souvent les études évaluatives se concentrent sur les résultats obtenus en termes de caractéristiques et de conduites des individus en oubliant complètement le processus de mise en œuvre des politiques. Or, en réalité, ce processus implique la mobilisation et la mise en synergie de multiples ressources. Comme le souligne l'auteur, " ce processus se déroule dans l'entreprise (avec ses différents acteurs internes) mais aussi à l'extérieur, avec de multiples acteurs. Ce sont les élus politiques, le service public de l'emploi, les administrations diverses qui interviennent dans le domaine, les associations et, bien sûr, les demandeurs d'emploi euxmêmes. Le processus comporte des phases complexes de coopération et de négociation entre acteurs, d'identification de besoins d'emploi, de définition de compétences, en liaison avec des choix financiers et organisationnels " (p. 286). L'examen de la mise en ouvre conduit à analyser la mise en place d'un partenariat avec les différents acteurs. Cet article est drôlement pertinent pour quiconque veut faire une analyse des déboires d'Emploi-Québec.

Un autre article, celui de Bernard Gazier, comporte des réflexions essentielles en ces temps où le phénomène des pénuries d'emplois semble permanent dans plusieurs pays. En effet, l'auteur discute des marchés transitionnels qui consistent en toutes les formes d'aménagement de l'emploi traditionnel visant à créer des transitions et des passerelles entre diverses positions sur le marché du travail. L'auteur insiste sur l'importance de développer des espaces sur le marché du travail où offreurs et demandeurs de tels statuts puissent se rencontrer. Contrairement au concept de revenu minimum garanti ou du principe de l'allocation universelle, les marchés transitionnels permettent de s'attaquer au problème de la répartition de la richesse collective sans pour autant " promouvoir une déconnexion délibérée entre le travail et le revenu, aux fins de développer la citoyenneté et la "liberté réelle" " (p. 348).

La conclusion de l'ouvrage, composée des articles de Jean-Claude Barbier et de Jérôme Gautié, bien que touffue, présente une critique des analyses de l'OCDE et cherche à offrir une alternative méthodologique aux analyses dites " universalistes » qui s'appuient sur des fondements théoriques qui remontent à Titmuss et qui visent à intégrer l'analyse sociétale de Maurice et alii ainsi que l'école de la régulation. On y discute abondamment de l'évolution des régimes d'emploi que l'on retrouve dans les modèles libéral, conservateur-corporatiste et social-démocrate. On y trouve une réflexion fort pertinente sur le Welfare to work qui tend à nuancer les diverses formes de réciprocité entre le bénéficiaire et le service public d'emploi que l'on adopte de plus en plus dans les pays européens et aux États-Unis.

En terminant, il convient de dire quelques mots sur les auteurs. Ceux-ci sont généralement des spécialistes du marché du travail et des pays présentés. Certains proviennent du milieu universitaire ou de centres de recherche alors que d'autres sont issus des milieux gouvernementaux. Les responsables de l'ouvrage auraient pu prévoir une présentation abrégée de chacun des auteurs.

Dans l'ensemble, les différentes contributions sont intéressantes et assez rigoureuses, mais parfois peuvent laisser sur leur appétit certains lecteurs... ce qui n'est pas nécessairement une mauvaise chose. En effet, elles attisent la curiosité et suscitent la réflexion sur des enjeux sociétaux telle la notion d'emploi qui, selon bon nombre d'auteurs de cet 
ouvrage, devrait ėtre conçue davantage comme le résultat d'un processus coopératif plutôt que d'une rencontre entre

DIANE BELLEMARF

offreurs et demandeurs.

Département de ressources humaines

et d'organisation

UQAM

\section{Flexibilité et créations d'emplois : un défi pour le dialogue social en Europe} par Hedva SARFATI, Paris et Montréal : l'Harmattan, 1999, 229 p., ISBN 2-7384$7722-4$

La hausse tendancielle des taux de chômage dans plusieurs pays depuis les années 1970 , ou leur stagnation à des niveaux jugés trop élevés, ainsi que la crainte de nouvelles flambées inflationnistes, ont amené une remise en question des politiques macro-économiques traditionnelles et favorisé un retour en force du credo néoclassique dans l'efficacité du libre jeu des forces du marché. Dans un contexte de concurrence accrue et de plus en plus internationale, la solution au chômage doit désormais passer par l'élimination des contraintes imposées à la variation des prix et à la mobilité des produits et de leurs facteurs de production. Dans le monde du travail, la recette se traduit par une plus grande capacité d'adaptation que doivent obtenir les entreprises dans l'utilisation et la rémunération de leur main-d'œuvre. Les stratégies de flexibilité du marché du travail devraient permettre à ces entreprises d'être plus compétitives et ainsi protéger, voire augmenter, les emplois. Ces stratégies visent une diminution des coûts de main-d'œuvre par une réduction des salaires et de certains avantages sociaux (flexibilités salariale et numérique) ou par une utilisation plus optimale du temps de travail (flexibilité numérique). La diminution des coûts de main-d'œuvre peut également être obtenue par une plus grande efficacité dans l'organisation de la production et du travail (flexibilité fonctionnelle). Cette dernière stratégie a reçu un accueil plus favorable des organisations syndicales dont plusieurs voyaient dans la réduction de la semaine de travail une des solutions au chômage endémique.

L'ouvrage d'Hedva Sarfati, politologue et ex-directrice du Département des re- lations professionnelles et de l'administration du travail au Bureau international du travail, questionne les mérites des stratégies de flexibilité du marché du travail pour une compétitivité accrue des entreprises et pour la protection et le développement de l'emploi. Son étude porte sur l'expérience de onze pays européens : l'Allemagne, l'Autriche, la Belgique, l'Espagne, la Finlande, la France, l'Irlande, l'Italie, les Pays-Bas, le Portugal et le Royaume-Uni. "Elle tente de démontrer que, dans cette difficile quête d'un équilibre entre flexibilité et sécurité, de meilleurs résultats ont pu être obtenus, pour les salariés comme pour les entreprises, dans les pays où le dialogue social existe ou a pu être mis en place, c'est-à-dire là où prévaut un climat de confiance entre les partenaires sociaux * (p. 7). Pour atteindre cet objectif, l'auteure s'est appuyée sur une revue des mesures législatives et des conventions collectives négociées à différents niveaux (national, sectoriel et d'entreprise) pour promouvoir l'emploi et réduire le chômage dans les pays étudiés.

L'étude comprend trois parties de deux chapitres chacune. Après une préface et une introduction qui se répètent sur certains aspects, la première partie de l'ouvrage ambitionne de retracer l'évolution du débat sur la flexibilité du marché du travail. Dans un premier chapitre, Hedva Sarfati situe le contexte de ce débat en s'appuyant sur le contenu de certaines études de l'OCDE et de la Commission européenne sur l'emploi et sur une enquête d'opinion réalisée par la Fondation européenne pour l'amélioration des conditions de vie au travail auprès des chefs d'entreprise sur la participation des salariés aux décisions. Elle 